\title{
BMJ Open Fruit and vegetable consumption and psychological distress: cross-sectional and longitudinal analyses based on a large Australian sample
}

\author{
Binh Nguyen, Ding Ding, Seema Mihrshahi
}

To cite: Nguyen B, Ding D, Mihrshahi S. Fruit and vegetable consumption and psychological distress: crosssectional and longitudinal analyses based on a large Australian sample. BMJ Open 2017;7:e014201.

doi:10.1136/bmjopen-2016014201

- Prepublication history for this paper is available online. To view these files please visit the journal online (http://dx.doi.org/10.1136/ bmjopen-2016-014201).

Received 8 September 2016 Revised 23 January 2017 Accepted 2 February 2017

CrossMark

Prevention Research Collaboration, Sydney School of Public Health, The University of Sydney, Camperdown, New South Wales, Australia

Correspondence to

Ms Binh Nguyen; thanh-binh.nguyen-duy@ sydney.edu.au

\section{ABSTRACT}

Objectives: Growing evidence suggests a link between diet and mental health. This study aimed to investigate the association between fruit and vegetable consumption and the prevalence and incidence of psychological distress in middle-aged and older Australians.

Design: Cross-sectional and prospective.

Setting: New South Wales, Australia.

Methods: A sample of 60404 adults aged $\geq 45$ years completed baseline (2006-2008) and follow-up (2010) questionnaires. Psychological distress was assessed at baseline and follow-up using the validated Kessler Psychological Distress Scale (K10), a 10-item questionnaire measuring general anxiety and depression. Psychological distress was defined as the presence of high-to-very high levels of distress (K10 score $\geq 22$ ). Usual fruit and vegetable consumption was assessed using short validated questions. The association between baseline fruit and vegetable consumption and the prevalence or incidence of psychological distress was examined using logistic regression models.

Results: At baseline, 5.6\% reported psychological distress. After a mean 2.7 years of follow-up, $4.0 \%$ of those who did not report distress at baseline reported distress at follow-up. Baseline fruit and vegetable consumption considered separately or combined, was associated with a lower prevalence of psychological distress even after adjustment for sociodemographic characteristics and lifestyle risk factors. Baseline fruit and vegetable consumption, measured separately or combined, was associated with a lower incidence of psychological distress in minimally adjusted models. Most of these associations remained significant at medium levels of intake but were no longer significant at the highest intake levels in fully adjusted models.

Conclusions: Increasing fruit and vegetable consumption may help reduce psychological distress in middle-aged and older adults. However, the association of fruit and vegetable consumption with the incidence of psychological distress requires further investigation, including the possibility of a threshold effect between medium and higher consumption levels.

\section{Strengths and limitations of this study}

- This study included a large sample size of 60404 participants for cross-sectional analyses and 54345 participants for longitudinal analyses.

- Analyses were adjusted for multiple sociodemographic and lifestyle-related covariates.

- The well-validated Kessler Psychological Distress Scale (K10) was used to assess psychological distress.

- The relatively short follow-up time may have been insufficient to observe the full extent of long-term associations between fruit and vegetable intake and psychological distress.

\section{INTRODUCTION}

There has been a global call for action by the WHO to make mental health a global development priority. ${ }^{1}$ Mental disorders affect a 10th of the world population and represent $30 \%$ of non-fatal global burden of disease. $^{2}$ Depression alone is a leading cause of disability worldwide ${ }^{3}$ and is projected to rank among the three leading causes of global disease burden by $2030 .^{4}$ There is an urgent call for public health strategies aimed at preventing the onset on common mental disorders, such as depression.

There has been considerable interest in the relationship between psychological wellbeing and lifestyle factors, with growing evidence for a link between mental health and diet. $^{5-7}$ The role of fruit and vegetables has received increasing attention, given evidence for its protective effects against chronic diseases such as cardiovascular disease and cancer. $^{8} 9$ Diets low in fruit have been recently identified as the leading dietary risk factor for global burden of disease. ${ }^{10}$

Findings from a recent meta-analysis, based on seven cross-sectional and four prospective studies, suggest that both fruit and 
vegetable consumption are significantly associated with a lower risk of depression. ${ }^{11}$ Several large cross-sectional studies have shown that greater consumption of fruit and vegetables is associated with better mental health, including lower odds of depression and psychological distress, in the general population. ${ }^{12-14}$ Fewer studies have investigated the longitudinal association between fruit and vegetable intake and depression. Higher consumption of fruit and/or vegetables was associated with lower odds of incident depression in middle-aged Australian women followed over 6 years, ${ }^{15}$ postmenopausal American women followed for 3 years ${ }^{16}$ and Spanish adults followed over 4 years. ${ }^{17}$ These findings are in agreement with previous cross-sectional and longitudinal studies that have found healthy dietary patterns, including high intakes of fruit and vegetables, to be associated with a lower risk of depression and anxiety, particularly in middle-aged and older adults. ${ }^{18-21}$

Depression in later life is associated with increased morbidity and mortality, and decreased physical, cognitive and social functioning. ${ }^{22}$ Improving mental health is an important public health challenge to address in an ageing population with a higher life expectancy. ${ }^{1}$ Therefore, the main objective of this study was to investigate the association between fruit and vegetable consumption and the prevalence and incidence of psychological distress in a large cohort of middle-aged and older Australians.

\section{METHODS}

\section{Study population}

The baseline data were from the Sax Institute's 45 and Up Study, a large-scale ( $n=267153$ ) population study of men and women aged 45 years and over, who were randomly sampled from the general population of New South Wales (NSW), Australia. From January 2006 to December 2008, eligible individuals joined the study by completing a postal questionnaire and providing written consent for participation and long-term follow-up. The 45 and Up Study has been described in detail elsewhere. ${ }^{23}$ A subsample of the 45 and Up Study was followed up in 2010 (ie, the Social, Economic, and Environmental Factor (SEEF) Study), with the first 100000 participants of the 45 and Up Study invited to complete the SEEF questionnaire $(60.4 \%$ response rate). A participant flow chart for this study is provided in figure 1. For cross-sectional analyses at baseline, the analytic sample included 60404 participants $(53.6 \%$ women). For longitudinal analyses, participants who reported on the baseline questionnaire that they had been treated for depression/anxiety in the previous month $(\mathrm{n}=3796)$, and/or taking antidepressant medication for most of the past 4 weeks $(n=700)$, and/or with high/very high levels of psychological distress $(n=3030$; defined as having a Kessler Psychological Distress Scale (K10) score $\geq 22^{24}$ were excluded $(\mathrm{n}=6067)$, leaving a final sample of 54345 participants.

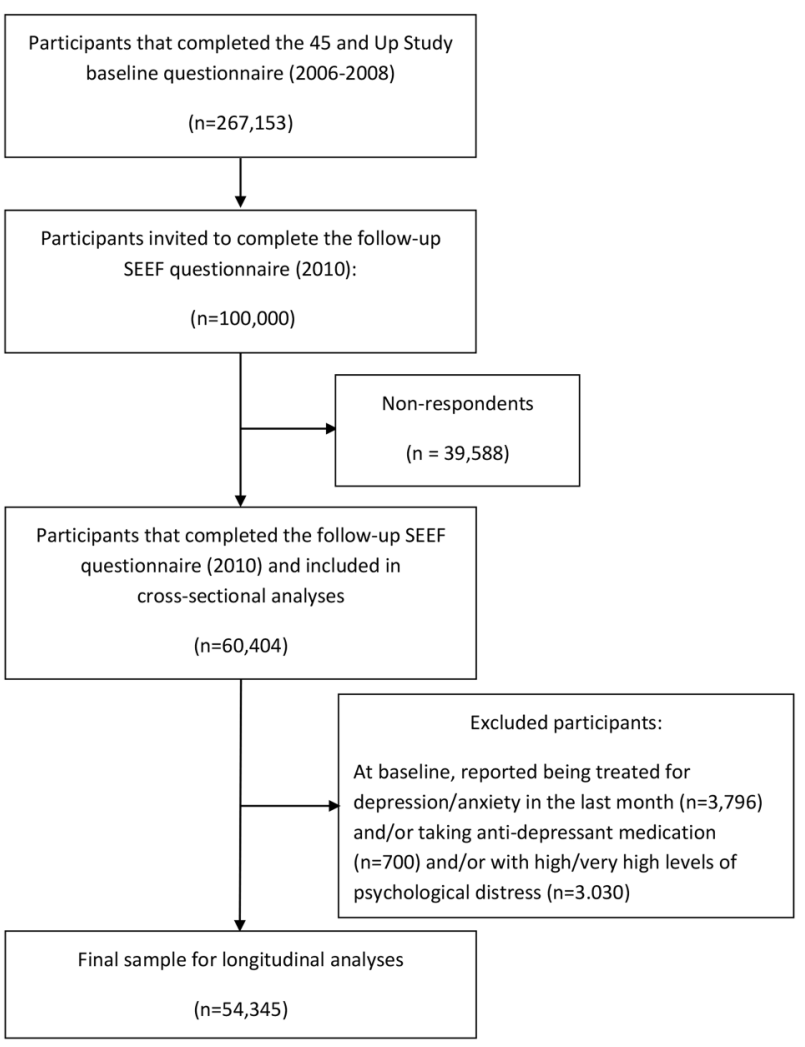

Figure 1 Participant flow chart. SEEF, Social, Economic, and Environmental Factor.

\section{Measurement}

The 45 and Up Study and SEEF Study questionnaires include questions on sociodemographic characteristics, personal and medical history, and lifestyle risk factors (available from http://www.saxinstitute.org.au/ our-work/45-up-study/questionnaires/).

\section{Outcome}

At both baseline and follow-up, participants' general level of psychological distress was assessed using the wellvalidated and widely used K10, a 10-item questionnaire about anxiety and depression symptoms experienced in the past 4 weeks. ${ }^{24} \mathrm{~A}$ five-point response scale (none of the time, a little of the time, some of the time, most of the time, all of the time) is used for each item, with scores ranging from 1 (none of the time) to 5 (all of the time). Scores to each question are added up to form the total K10 score, with a possible range of 10-50. For this study, score groupings and categories of psychological distress routinely used by the Australian Bureau of Statistics for national health surveys were adopted with total scores of: 10-15, 16-21, 22-29 and 30-50 indicating low, moderate, high and very high levels of psychological distress, respectively.

High K10 scores are strongly correlated with current WHO's Composite International Diagnostic Interview (CIDI) diagnosis of anxiety and affective disorders. ${ }^{24}$ Prevalence of psychological distress at baseline was 
defined as the presence of high-to-very high levels of psychological distress (K10 score $\geq 22$ ). Incidence of psychological distress was defined as: (1) not being treated for anxiety/depression in the previous month, and/or not taking antidepressant medication for most of the past 4 weeks, and/or not having high/very high levels of psychological distress (K10 score <22) at baseline, and (2) the presence of high-to-very high levels of psychological distress (K10 score $\geq 22)$ at follow-up. Psychological distress was treated as binary outcome variable in the analyses (K10 score $<22$ vs $\geq 22$; ie, low-to-moderate vs high-to-very high levels of distress).

\section{Exposure}

Usual fruit and vegetable consumption was assessed at baseline using the following validated short questions commonly used in health monitoring and surveillance: ${ }^{25}$

1. 'About how many serves of fruit do you usually have each day?' One serve of fruit was defined as one medium piece or two small pieces of fresh fruit, or one cup of diced or canned fruit pieces.

2. 'About how many serves of vegetables do you usually eat each day?' One serve of vegetables was defined as half a cup of cooked vegetables (including potatoes) or one cup of raw vegetables (eg, salad).

Total fruit and vegetable consumption was derived by summing the reported number of fruit and vegetables consumed daily. Fruit and vegetable consumption, considered separately and combined, was categorised into tertiles. Using quantiles ensures that the range in exposure is captured evenly across distribution categories, which facilitates comparison between different levels of fruit and vegetable consumption among the study cohort, and has been previously used in another large cohort study. ${ }^{16}$

\section{Covariates}

Covariates included baseline self-reported sociodemographic characteristics such as sex, age, highest level of education ( $\leq 10$ years of schooling, high school/trade apprenticeship/certificate/diploma, university degree/ higher), marital status (married/living with a partner vs single/widowed/divorced/separated), household annual income ( $<\$ 30000, \$ 30000-\$ 69999, \geq \$ 70000$, would rather not answer this question), self-reported history of major chronic disease (cancer other than nonmelanoma skin cancer, cardiovascular disease (heart disease, stroke or blood clot), diabetes or hypertension; yes vs no) and the following lifestyle risk factors: body mass index (BMI; derived from self-reported height and weight; defined as underweight $\left(\left(<18.5 \mathrm{~kg} / \mathrm{m}^{2}\right)\right.$, normal weight (18.5 to $\left.<25 \mathrm{~kg} / \mathrm{m}^{2}\right)$, overweight/obese $\left.\left(\geq 25 \mathrm{~kg} / \mathrm{m}^{2}\right)\right)$, alcohol intake $(\leq 14$ or $>14$ drinks/ week), smoking status (current regular smoker vs not currently a regular smoker) and physical activity levels (assessed using validated questions from the Active Australia Survey, ${ }^{26}$ categorised as $<150,150-299$ and $\geq 300 \mathrm{~min} /$ week).

\section{Statistical analysis}

The association between baseline fruit and vegetable consumption and the prevalence/incidence of psychological distress (K10 score $\geq 22$ ) was examined using logistic regression models. ORs with 95\% CIs are presented for unadjusted, age-adjusted and sex-adjusted, and models adjusted for all covariates as described above. We tested effect modification by sex by fitting interaction terms. To examine potential sex differences, the analyses were further stratified by sex. If 1 out of 10 responses to K10 questions was missing (for $3.2 \%$ and $2.8 \%$ of participants included in cross-sectional and longitudinal analyses, respectively), the missing value was imputed using the mean score across the other 9 questions. ${ }^{27}$ If more than one response was missing, K10 scores were considered as missing. $p$ Values $<0.05$ were considered statistically significant. All analyses were conducted using SPSS V.22 (IBM Corp, Armonk, New York, USA).

\section{RESULTS}

\section{Participant characteristics}

Table 1 shows baseline participant characteristics based on K10 score at follow-up. Overall, the mean age (SD) of participants was 62.2 (10.6) years, more than half $(53.6 \%)$ were women, over a quarter $(26 \%)$ had a university degree/higher, over three-quarters $(78 \%)$ were in a married/de facto relationship, and a quarter $(25.7 \%)$ reported a household annual income $\geq \$ 70000$. The mean (SD) serves of fruit and vegetables were, respectively, 2.0 (1.4) and 3.9 (2.6) serves/day. The average follow-up time period was 2.7 (0.9) years. Compared with men, women were more likely to be younger, less educated, single/widowed/divorced/separated, have a lower household annual income, a lower BMI, and to consume more fruit and vegetables and less alcohol. Participants with high-to-very high levels of psychological distress $(5.6 \%)$ at baseline were more likely to be women, relatively younger, less educated and have a lower household annual income. These participants were also more likely to: have a higher BMI, be a current smoker, be less physically active and have a history of chronic disease.

\section{Prevalence of psychological distress}

The ORs for the association between separate or combined fruit and vegetable consumption and the prevalence of high-to-very high levels of psychological distress $(\mathrm{K} 10 \geq 22)$ are presented in table 2. Consumption of fruit and vegetables, considered separately or combined, was consistently associated with a lower prevalence of psychological distress. Following adjustment for all covariates, these associations were slightly attenuated compared with the unadjusted model but remained significant. Other covariates which were significantly associated with the prevalence of psychological distress were being relatively younger, single/divorced/widowed/separated, a 
Table 1 Baseline characteristics of participants according to sex and K10 score at baseline $(n=60404 ; 2006-2010)^{*}$

\begin{tabular}{|c|c|c|c|c|c|c|}
\hline \multirow[b]{2}{*}{ Variable } & \multirow[b]{2}{*}{ All } & \multirow[t]{2}{*}{ Men } & \multirow{2}{*}{ Women } & \multicolumn{3}{|c|}{ K10 score at baseline† } \\
\hline & & & & $<22$ & $\geq 22$ & p Value \\
\hline Sample size & 60404 & 28057 & 32347 & 51393 & 3030 & \\
\hline Mean (SD) follow-up time (years) & $2.67(0.93)$ & $2.67(0.93)$ & $2.68(0.94)$ & $2.67(0.94)$ & $2.72(0.95)$ & 0.009 \\
\hline Women (\%) & 53.6 & - & - & 53.3 & 56.2 & $<0.001$ \\
\hline Mean (SD) age (years) & $62.2(10.6)$ & $63.9(10.7)$ & $60.8(10.2) \S$ & $61.6(10.3)$ & $58.6(9.6)$ & $<0.001$ \\
\hline Highest education§ (\%) & & & & & & $<0.001$ \\
\hline University and higher & 26.2 & 28.0 & 24.7 & 28.3 & 20.0 & \\
\hline High school/trade apprenticeship/certificate/Diploma & 42.7 & 48.5 & 37.7 & 43.3 & 41.3 & \\
\hline$\leq 10$ years & 31.1 & 23.4 & 37.6 & 28.4 & 68.1 & \\
\hline Married/living with a partner (\%) & 78.0 & 83.5 & $73.2 \S$ & 79.6 & 68.1 & $<0.001$ \\
\hline Household annual income§ (\%) & & & & & & $<0.001$ \\
\hline$<\$ 30000$ & 29.5 & 28.5 & 30.4 & 26.7 & 43.4 & \\
\hline$\$ 30000-\$ 69999$ & 28.9 & 31.0 & 27.0 & 29.8 & 25.4 & \\
\hline$\geq \$ 70000$ & 25.7 & 29.3 & 22.6 & 28.1 & 16.7 & \\
\hline Did not specify & 15.9 & 11.2 & 20.0 & 15.4 & 14.4 & \\
\hline BMI category§ (\%) & & & & & & $<0.001$ \\
\hline Underweight $\left(<18.5 \mathrm{~kg} / \mathrm{m}^{2}\right)$ & 1.2 & 0.7 & 1.7 & 1.1 & 2.2 & \\
\hline Normal weight $\left(18.5\right.$ to $\left.<25 \mathrm{~kg} / \mathrm{m}^{2}\right)$ & 37.9 & 31.8 & 43.3 & 38.4 & 31.0 & \\
\hline Overweight or obese $\left(\geq 25 \mathrm{~kg} / \mathrm{m}^{2}\right)$ & 60.9 & 67.6 & 55.0 & 60.5 & 66.8 & \\
\hline Current smoker (\%) & 5.7 & 5.7 & 5.7 & 5.3 & 13.9 & $<0.001$ \\
\hline Usually consumes $>14$ alcohol drinks/week & 14.9 & 24.7 & $6.3 \S$ & 15.3 & 14.8 & 0.44 \\
\hline Mean (SD) fruit consumption (serves/day) & $2.0(1.4)$ & $1.9(1.5)$ & $2.2(1.4) \S$ & $2.0(1.4)$ & $1.9(1.5)$ & $<0.001$ \\
\hline Mean (SD) vegetable consumption (serves/day) & $3.9(2.6)$ & $3.4(2.6)$ & $4.4(2.6) \S$ & $3.9(2.6)$ & $3.7(2.7)$ & $<0.001$ \\
\hline Physical activity level (\%) & & & & & & $<0.001$ \\
\hline$<150 \mathrm{~min} /$ week & 18.9 & 19.2 & 18.8 & 17.5 & 28.0 & \\
\hline 150-299 min/week & 16.6 & 16.4 & 16.9 & 16.6 & 18.7 & \\
\hline$\geq 300 \mathrm{~min} /$ week & 64.4 & 64.5 & 64.4 & 65.9 & 53.4 & \\
\hline History of chronic disease (\%) & 51.8 & 56.5 & $47.8 \S$ & 50.9 & 54.0 & $<0.001$ \\
\hline $\begin{array}{l}\text { *Data are presented as means (SD) or percentages (\%). } \\
\text { †The total K10 score is based on a } 10 \text {-item questionnaire abo } \\
\text { Participants were grouped according to K10 scores and categ } \\
\text { psychological distress }(\geq 22) \text {. K10 data were missing for } n=598 \\
\text { tp Value from independent t-tests for continuous variables an } \\
\S \text { Significantly different from men (all p<0.001). } \\
\text { BMI, body mass index; K10, Kessler Psychological Distress S }\end{array}$ & $\begin{array}{l}\text { out anxiety an } \\
\text { orised as at "I } \\
81 \text {. } \\
\text { id from } \chi^{2} \text { test } \\
\text {. }\end{array}$ & $\begin{array}{l}\text { d depression } \\
\text { ow-to-modera }\end{array}$ & $\begin{array}{l}\text { symptoms expe } \\
\text { te risk' (K10<22 } \\
\text { cal variables. }\end{array}$ & $\begin{array}{l}\text { erienced in } \\
\text { 2) or at 'hig }\end{array}$ & $\begin{array}{l}\text { past } 4 \text { weeks } \\
\text { o-very high ris }\end{array}$ & $\begin{array}{l}\text { ss. } \\
\text { sk' of }\end{array}$ \\
\hline
\end{tabular}

current smoker, lower education, lower household annual income, lower BMI, low physical activity levels and a self-reported history of chronic disease. There was a significant interaction between combined fruit and vegetable consumption and sex $(\mathrm{p}=0.049)$. When analyses were stratified by sex (table 3 ), the association between fruit and vegetable consumption, measured separately or combined, and the prevalence of psychological distress was markedly stronger in women and was significant for all consumption tertiles $(p \leq 0.001)$. Among men, only those in the medium tertiles of separate fruit and vegetable consumption had significantly lower odds of psychological distress.

\section{Incidence of psychological distress}

After an average of 2.7 years of follow-up, $4.0 \%$ of those who did not report distress at baseline reported distress at follow-up. Table 4 shows the association between fruit and vegetable consumption and the incidence of high-to-very high levels of psychological distress (K10 $\geq 22)$. Similar to cross-sectional findings, fruit and vegetable consumption, measured separately or combined, was significantly associated with a lower incidence of psychological distress in unadjusted and minimally adjusted models. In the fully adjusted models, the medium tertiles of combined fruit and vegetable consumption, and separate vegetable consumption, remained significantly associated with reduced odds of psychological distress. The association between the medium tertile of fruit consumption and the incidence of psychological distress approached significance $(p=0.07)$. However, the association between the highest tertile of consumption and the incidence of psychological distress did not remain significant for consumption of fruit and vegetables considered either separately or combined. Other covariates which were significantly associated with the incidence of psychological distress were being relatively younger, single/divorced/ widowed/separated, a current smoker, lower education, lower household annual income, lower alcohol intake, lower BMI, low physical activity levels and a self-reported history of chronic disease. The interaction between 


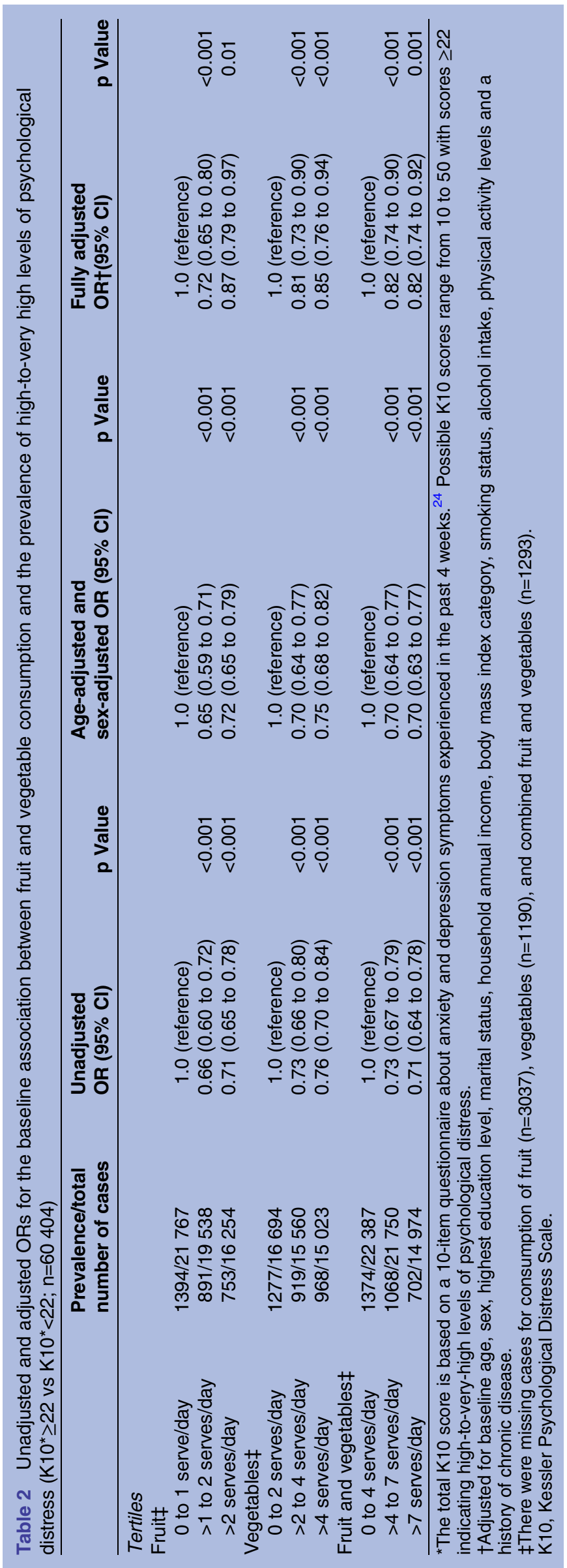

combined fruit and vegetable consumption and sex approached significance $(p=0.08)$. When analyses were stratified by sex (table 3), the association between fruit and vegetable consumption, considered separately or combined, and the incidence of psychological distress was stronger in women and significant for all consumption tertiles except for the highest fruit $(p=0.06)$, vegetable $(p=0.17)$, and combined fruit and vegetable tertiles $(p=0.09)$ in the fully adjusted models. There was no significant association between consumption of fruit and vegetables and the incidence of psychological distress in men.

\section{DISCUSSION}

In this large cohort of middle-aged and older Australian adults, consumption of fruit and vegetables was significantly associated with the prevalence of psychological distress even after accounting for sociodemographic characteristics and other lifestyle risk factors. The longitudinal associations with psychological distress were less consistent. The association between fruit and vegetable intake and the incidence of psychological distress was significant after accounting for age and sex. After adjustment for all possible confounders, while this association remained mostly significant at medium levels of intake, it did not remain significant at the highest levels of intake. When considered separately in each sex, the association of fruit and vegetable consumption with either the prevalence or incidence of psychological distress was stronger in women, with no clear associations with the incidence of psychological distress in men.

Findings in this study are generally in agreement with those from a recent meta-analysis, based on seven crosssectional and four cohort studies, which has found separate fruit and vegetable consumption to be inversely associated with the risk of depression. ${ }^{11}$ Although findings from individual cross-sectional and prospective studies were mixed, in subgroup analysis by study design, the meta-analysis showed significant associations in crosssectional and prospective studies for fruit intake, and in prospective studies only for vegetable intake. In relation to combined fruit and vegetable consumption, several large cross-sectional studies have also demonstrated significant inverse associations with psychological wellbeing, even after accounting for multiple covariates. ${ }^{12-14}$ A positive association between combined fruit and vegetable consumption and well-being, assessed using seven different measures of mental health, was shown in three separate data sets, which together involved 80000 randomly selected British adults. ${ }^{12}$ In a repeated crosssectional study of 296121 Canadians with five waves of a national, population-based survey, lower odds of depression and psychological distress were consistently associated with greater combined fruit and vegetable consumption. ${ }^{13}$ Our cross-sectional findings are also in line with those from a recent population-based Swiss survey of 20220 individuals, which found that daily 
Table 3 Adjusted ORs for the prevalence and incidence of high-to-very high levels of psychological distress (K10* $\geq 22$ vs $K 10^{*}<22$ ) by baseline fruit and vegetable consumption and stratified by sex

Cross-sectional analysis

Male

Female

Unadjusted OR Age-adjusted Fully adjusted

Age-adjusted Fully adjusted Unadjusted p Value OR $(95 \% \mathrm{Cl}) \quad$ p Value OR† $(95 \% \mathrm{Cl}) \quad$ p Value OR $(95 \% \mathrm{Cl})$

p Value OR $(95 \% \mathrm{Cl})$

p Value ORt $(95 \% \mathrm{Cl})$

p Value

Tertiles

Fruit

0 to 1 serve/day 1.0 (reference)

$>1$ to 2 serves/day 0.69 ( 0.59 to 0.79$)$

$>2$ serves/day $\quad 0.80(0.69$ to 0.93$)$ Vegetables

0 to 2 serves/day 1.0 (reference)

$>2$ to 4 serves/day $0.70(0.61$ to 0.80$)$

$>4$ serves/day $0.82(0.71$ to 0.95$)$

Fruit and vegetables

0 to 4 serves/day 1.0 (reference)

$>4$ to 7 serves/day 0.80 ( 0.70 to 0.91$)$

$>7$ serves/day $\quad 0.79(0.67$ to 0.93$)$

\begin{tabular}{|c|c|c|c|}
\hline & 1.0 (reference) & & 1.0 (reference) \\
\hline$<0.001$ & 0.71 (0.61 to 0.82 ) & $<0.001$ & 0.78 (0.67 to 0.91 ) 0.002 \\
\hline 003 & 0.83 (0.72 to 0.96$)$ & 0.02 & 0.99 (0.84 to 1.17$) 0.95$ \\
\hline
\end{tabular}

1.0 (reference)

1.0 (reference)

1.0 (reference)

0.61 (0.54 to 0.68$)<0.001 \quad 0.65(0.57$ to 0.73$)<0.001 \quad 0.79$ (0.69 to 0.91$) \quad 0.001$

1.0 (reference) 1.0 (reference)

$\begin{array}{lllllllllll}0.007 & 0.88(0.76 \text { to } 1.01) & 0.07 & 0.91(0.78 \text { to } 1.06) & 0.23 & 0.64(0.57 \text { to } 0.72)<0.001 & 0.68(0.60 \text { to } 0.77)<0.001 & 0.80(0.70 \text { to } 0.92) & 0.001\end{array}$

1.0 (reference) $\quad 1.0$ (reference)

$\begin{array}{lllll}0.001 & 0.82(0.72 \text { to } 0.94) & 0.003 & 0.92(0.80 \text { to } 1.07) & 0.28\end{array}$

1.0 (reference) $\quad 1.0$ (reference) 1.0 (reference)

Longitudinal analysis $¥$

\begin{tabular}{|c|c|c|c|c|c|c|c|}
\hline Male & & & & Female & & & \\
\hline $\begin{array}{l}\text { Jnadjusted OR } \\
95 \% \mathrm{Cl})\end{array}$ & $\begin{array}{l}\text { Age-adjusted } \\
\text { p Value OR }(95 \% \mathrm{Cl})\end{array}$ & $\begin{array}{l}\text { Fully adjusted } \\
\text { p Value ORt }(95 \% \mathrm{Cl})\end{array}$ & p Value & $\begin{array}{l}\text { Unadjusted } \\
\text { OR }(95 \% \mathrm{Cl})\end{array}$ & $\begin{array}{l}\text { Age-adjusted } \\
\text { p Value } \mathrm{OR}(95 \% \mathrm{Cl})\end{array}$ & p Value & $\begin{array}{l}\text { Fully adjusted } \\
\text { ORt }(95 \% \mathrm{Cl})\end{array}$ \\
\hline
\end{tabular}

\begin{tabular}{|c|c|c|c|c|c|c|c|c|c|c|c|c|}
\hline \multicolumn{13}{|l|}{ Tertiles } \\
\hline \multicolumn{13}{|l|}{ Fruit } \\
\hline 0 to 1 serve/day & 1.0 (reference) & & 1.0 (reference) & & 1.0 (reference) & & 1.0 (reference) & & 1.0 (reference) & & 1.0 (reference) & \\
\hline$>1$ to 2 serves/day & 0.87 (0.74 to 1.02$)$ & 0.09 & 0.87 (0.74 to 1.02$)$ & 0.09 & $0.95(0.80$ to 1.13$)$ & 0.56 & 0.72 (0.62 to 0.83$)$ & $<0.001$ & $0.72(0.62$ to 0.84$)$ & $<0.001$ & $0.84(0.71$ to 1.0$)$ & 0.04 \\
\hline$>2$ serves/day & $0.90(0.75$ to 1.06$)$ & 0.21 & 0.89 (0.75 to, 1.06$)$ & 0.20 & $0.98(0.81$ to 1.19$)$ & 0.85 & 0.68 (0.58 to 0.80$)$ & $<0.001$ & 0.69 (0.59 to 0.81$)$ & $<0.001$ & $0.84(0.70$ to 1.0$)$ & 0.06 \\
\hline \multicolumn{13}{|l|}{ Vegetables } \\
\hline 0 to 2 serves/day & 1.0 (reference) & & 1.0 (reference) & & 1.0 (reference) & & 1.0 (reference) & & 1.0 (reference) & & 1.0 (reference) & \\
\hline$>2$ to 4 serves/day & $0.86(0.73$ to 1.0$)$ & 0.05 & $0.85(0.73$ to 1.0$)$ & 0.05 & $0.94(0.79$ to 1.11$)$ & 0.45 & 0.67 (0.57 to 0.78$)$ & $<0.001$ & $0.67(0.57$ to 0.78$)$ & $<0.001$ & 0.82 (0.69 to 0.98$)$ & 0.03 \\
\hline$>4$ serves/day & $0.96(0.81$ to 1.13$)$ & 0.59 & 0.95 (0.81 to 1.12$)$ & 0.55 & 0.94 (0.78 to 1.13$)$ & 0.51 & 0.75 (0.65 to 0.87$)$ & $<0.001$ & $0.76(0.65$ to 0.88$)$ & $<0.001$ & 0.89 (0.75 to 1.05$)$ & 0.17 \\
\hline \multicolumn{13}{|c|}{ Fruit and vegetables } \\
\hline 0 to 4 serves/day & 1.0 (reference) & & 1.0 (reference) & & 1.0 (reference) & & 1.0 (reference) & & 1.0 (reference) & & 1.0 (reference) & \\
\hline$>4$ to 7 serves/day & $0.88(0.76$ to 1.03$)$ & 0.11 & $0.88(0.76$ to 1.03$)$ & 0.11 & $0.98(0.83$ to 1.16$)$ & 0.82 & 0.63 (0.55 to 0.73$)$ & $<0.001$ & $0.64(0.55$ to 0.74$)$ & $<0.001$ & 0.77 (0.65 to 0.91$)$ & 0.002 \\
\hline$>7$ serves/day & $0.96(0.80$ to 1.14$)$ & 0.62 & 0.95 (0.79 to 1.14$)$ & 0.57 & 0.94 (0.77 to 1.15$)$ & 0.52 & 0.71 (0.61 to 0.83 ) & $<0.001$ & $0.72(0.62$ to 0.84$)$ & $<0.001$ & 0.86 (0.72 to 1.02$)$ & 0.09 \\
\hline
\end{tabular}

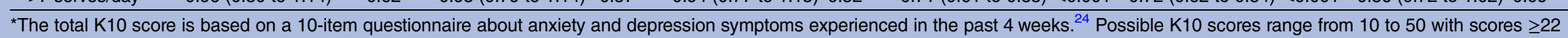
indicating high-to-very-high levels of psychological distress.

†Adjusted for baseline age, sex, highest education level, marital status, household annual income, body mass index category, smoking status, alcoholic intake, physical activity levels and a history of chronic disease.

$\S$ Participants who reported having been recently treated for depression/anxiety and/or taking antidepressant medication and/or with a K10 score $\geq 22$ ( $n=6067$ ) at baseline were excluded from longitudinal analyses.

K10, Kessler Psychological Distress Scale. 


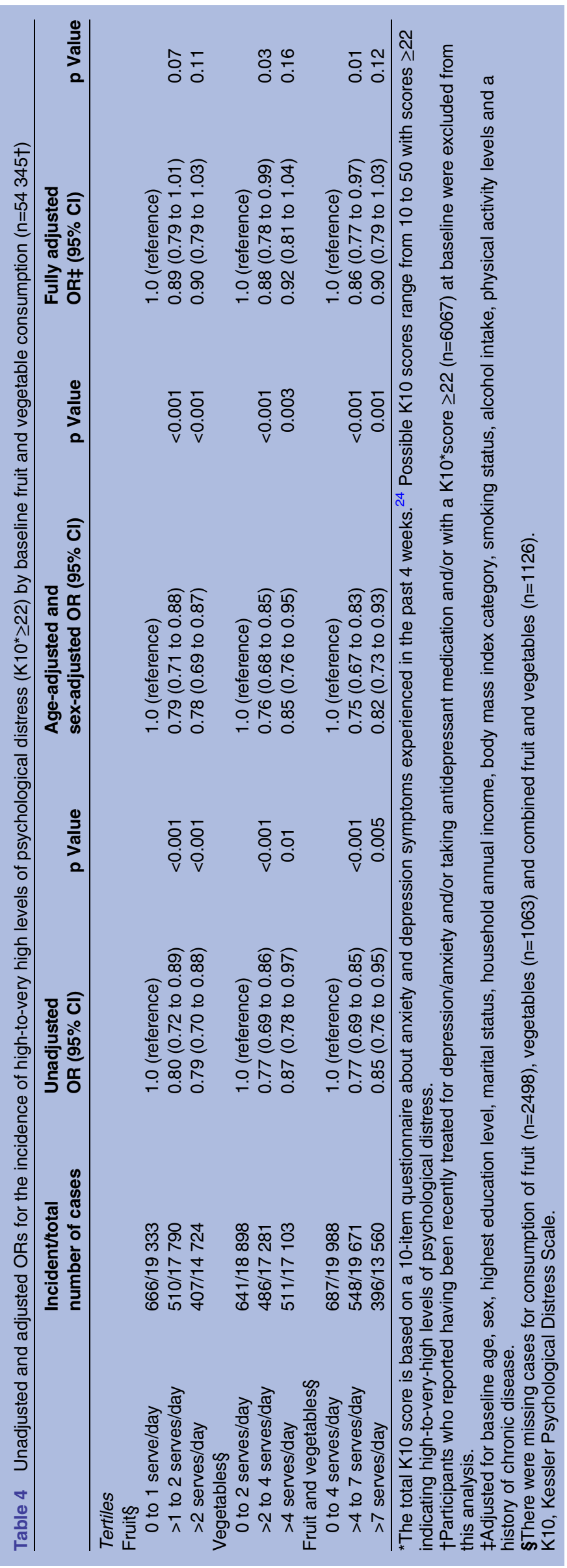

recommended intake of five servings of fruit and vegetables was associated with a lower likelihood of high and moderate psychological distress. ${ }^{14}$

Our longitudinal findings add to the limited evidence base for an association between fruit and vegetable consumption and the incidence of psychological distress. Although longitudinal associations with psychological distress did not remain significant at higher levels of fruit and vegetable intake, the direction of these associations was in agreement with findings from previous studies. Among the few prospective studies which have examined the relationship between fruit and vegetable intake and the incidence of depression, mostly in similar-aged samples, ${ }^{15-17} 28$ all but one $\operatorname{study}^{28}$ have shown significant protective effects of fruit ${ }^{15} 17$ or both fruit and vegetables. ${ }^{16}$ A recent study involving a nationally representative sample of 12385 Australian adults surveyed over several years reported that combined fruit and vegetable consumption was predictive of increased happiness, life satisfaction and well-being, with improvements observed within 2 years. ${ }^{29}$ In the case of our study, the longitudinal association between fruit and vegetable consumption and psychological distress was attenuated the most between the age-adjusted and sex-adjusted model and the fully adjusted model, suggesting confounding. This may indicate that those who consume healthy amounts of fruit and vegetables are more likely to have favourable socioeconomic status and other lifestyle risk factors (eg, physical activity), which together contributed to lower psychological distress.

This study is among the first to report associations between fruit and vegetable consumption and psychological well-being separately for men and women. Sex was a significant effect modifier of the association between fruit and vegetable consumption and psychological distress. We found that fruit and vegetables were more protective for women than men, suggesting that women may be more responsive to the effects of fruit and vegetables. It is possible that there may be a true physiological difference between men and women, although a mechanism that could explain this difference remains unclear, or perhaps women more accurately report consumption of fruit and vegetables than men. However, these preliminary findings need to be confirmed by additional studies.

Future investigations should also explore the possibility of a threshold between medium and higher consumption levels. In our study, fruit and vegetable consumption at the highest levels was not protective against psychological distress in fully adjusted models, suggesting a potential threshold effect. This was also evident in the fully adjusted models in the cross-sectional analysis in men, and the longitudinal analysis in women. The reason for this observation is unknown. It is possible that consuming more fruits and vegetables beyond the potential threshold is no longer beneficial. However, the observed pattern of association could also be a result of residual confounding. For 
example, participants consuming higher amounts of fruit and vegetables may also have been consuming larger quantities of other foods which could lead to psychological distress. However, despite adjusting for BMI in our analyses, this study did not measure other potential dietary confounders. The study's findings also did not change when adjusting for BMI as a continuous variable rather than a categorical variable. Participants with very high fruit and vegetable consumption may have other unmeasured characteristics that could have offset the beneficial effects of fruit and vegetable consumption. Finally, it is important to acknowledge that fruit and vegetable consumption was based on a one-time measure only, which could not take into account longterm consumption patterns. However, as compared with baseline, we found a similar pattern of consumption at follow-up $(93 \%$ of participants remained in the same consumption categories between baseline and follow-up). Some of these limitations should be addressed in future studies.

Although these remain to be elucidated, several mechanisms may underlie the relationship between high fruit and vegetable consumption and greater psychological well-being. ${ }^{30}$ Fruit and vegetables are rich in micronutrients and phytochemicals that may help reduce oxidative stress and inflammation, processes that can have detrimental effects on mental health. For example, antioxidants such as vitamins $\mathrm{C}, \mathrm{E}$ and polyphenols may help reduce oxidative stress while the mineral magnesium has been associated with lower levels of $\mathrm{C}$ reactive protein, a marker of low-grade inflammation. ${ }^{30}$ Deficiencies in B vitamins such as folic acid (vitamin $\mathbf{B}_{9}$ ) have been associated with depression. ${ }^{31}$ Low levels of these vitamins can cause high homocysteine levels which in turn can impair methylation processes involved in the synthesis and metabolism of neurotransmitters that may affect mood. ${ }^{32}$

\section{Strengths and limitations}

This study had several strengths including a large sample size, a prospective design and the inclusion of multiple sociodemographic and lifestyle-related covariates and the use of the well-validated K10 to assess psychological distress. High K10 scores are strongly correlated with CIDI diagnoses of anxiety and depression. ${ }^{24}$ Several study limitations should be noted. The follow-up period may have been too short to observe the full extent of long-term associations between fruit and vegetable intake and psychological distress. Although the assessment of fruit and vegetable consumption was based on short validated questions, this assessment method may be prone to reporting bias. In addition, the assessment of dietary intake was not detailed and limited to a few questions only. There may be residual confounding from unmeasured dietary confounders including total energy intake and other potential confounders such as illicit drug use, a history of mental illness and unmeasured cardiometabolic components, despite adjustment for multiple covariates. Although data were available for fish consumption, another potential dietary confounder, this variable was not included as a covariate due to the lack of variance observed ('yes/no' question for ever consumption of fish only) and adjusting for fish consumption in our analyses also did not change our results. Further, the possibility of reverse causation (ie, that depression leads to poor diet including inadequate fruit and vegetable consumption) could not be eliminated, but was reduced by excluding participants being treated for depression/anxiety, taking antidepressant medication or who reported high-to-very levels of psychological distress at baseline from the longitudinal analyses. Several prospective cohort studies have not found evidence for reverse causation, with diet quality related to subsequent mental health but baseline mental health not associated with subsequent diet quality. ${ }^{15} \quad 17 \quad 21$ However, a recent nationally representative longitudinal study of Canadians, which explicitly tested reverse causation, showed that the association between fruit and vegetable consumption, other health behaviours and depressive symptoms are complex and bi-directional and warrants further investigation. ${ }^{33}$

\section{CONCLUSIONS}

Fruit and vegetable consumption may help reduce the prevalence of psychological distress among middle-aged and older adults. However, the association between fruit and vegetable consumption and the incidence of psychological distress requires further investigation and possibly, a longer follow-up time. Fruit and vegetable consumption may help reduce psychological distress among middle-aged and older females in a crosssectional context, but not potentially at the highest levels of intake in females over time. Consumption at medium levels of intake may help lower psychological distress in men in a cross-sectional context; however, longitudinal associations remain unclear. Although findings from this study lend support to existing public health guidelines which encourage fruit and vegetable consumption as part of a healthy diet and add evidence to support the benefits of fruit and vegetables for mental health, further research is clearly needed.

Acknowledgements This research was completed using data collected through the 45 and Up Study (http://www.saxinstitute.org.au). The 45 and Up Study is managed by the Sax Institute in collaboration with major partner Cancer Council NSW; and partners: the National Heart Foundation of Australia (NSW Division); NSW Ministry of Health; NSW Government Family and Community Services-Ageing, Carers and the Disability Council NSW; and the Australian Red Cross Blood Service. The authors thank the many thousands of people participating in the 45 and Up Study and the SEEF Study. The SEEF research was funded by the National Health and Medical Research Council (NHMRC) Strategic Award for Preventive Healthcare and Strengthening Australia's Social Economic Fabric (the SEEF Project; ID: 402810).

Contributors BN, DD and SM participated in the design of the study. BN carried out the statistical analyses. BN, DD and SM helped draft the manuscript. All authors helped with the interpretation of the data and revised the manuscript critically for important intellectual content. All authors read and approved the manuscript. 
Funding This research was funded from a Development Award from the Cardiovascular Research Network of NSW. BN was supported by an Australian Postgraduate Award and a University of Sydney Merit Award. DD was funded by an Early Career Fellowship from the NHMRC of Australia (reference number 1072223).

\section{Competing interests None declared.}

Ethics approval The 45 and Up Study was granted ethical approval by the University of NSW Human Research Ethics Committee (reference HREC 05035/HREC 10186) and the SEEF Study by the University of Sydney Human Research Ethics Committee (reference 10-2009/12187).

Provenance and peer review Not commissioned; externally peer reviewed.

Data sharing statement No additional data are available.

Open Access This is an Open Access article distributed in accordance with the Creative Commons Attribution Non Commercial (CC BY-NC 4.0) license, which permits others to distribute, remix, adapt, build upon this work noncommercially, and license their derivative works on different terms, provided the original work is properly cited and the use is non-commercial. See: http:// creativecommons.org/licenses/by-nc/4.0/

\section{REFERENCES}

1. World Health Organization. Out of the shadows: making mental health a global development priority. 2016. http://www.who.int/ mediacentre/events/2016/mental-health-meeting/en/

2. Patel V, Saxena S. Transforming lives, enhancing communitiesinnovations in global mental health. NEJM 2014;370:498-501.

3. Whiteford HA, Ferrari AJ, Degenhardt L, et al. The global burden of mental, neurological and substance use disorders: an analysis from the Global Burden of Disease Study. PLOS ONE 2015;10:e0116820.

4. Mathers CD, Loncar D. Projections of global mortality and burden of disease from 2002 to 2030. PLOS Med 2006;3:e442.

5. Appleton KM, Rogers PJ, Ness AR. Updated systematic review and meta-analysis of the effects of $n-3$ long-chain polyunsaturated fatty acids on depressed mood. Am J Clin Nutr 2010;91:757-70.

6. Lai JS, Hiles S, Bisquera A, et al. A systematic review and meta-analysis of dietary patterns and depression in community-dwelling adults. Am J Clin Nutr 2014;99:181-97.

7. Skarupski KA, Tangney $\mathrm{C}$, $\mathrm{Li} \mathrm{H}$, et al. Longitudinal association of vitamin B-6, folate, and vitamin B-12 with depressive symptoms among older adults over time. Am J Clin Nutr 2010;92:330-5.

8. Wang $\mathrm{X}$, Ouyang $\mathrm{Y}$, Liu J, et al. Fruit and vegetable consumption and mortality from all causes, cardiovascular disease, and cancer: systematic review and dose-response meta-analysis of prospective cohort studies. BMJ 2014;349:94490.

9. World Cancer Research Fund and the American Institute for Cancer Research. Food, nutrition, physical activity, and the prevention of cancer: a global perspective. Washington DC: American Institute for Cancer Research, 2007.

10. Lim SS, Vos T, Flaxman AD, et al. A comparative risk assessment of burden of disease and injury attributable to 67 risk factors and risk factor clusters in 21 regions, 1990-2010: a systematic analysis for the Global Burden of Disease Study 2010. Lancet 2012;380:2224-60.

11. Liu X, Yan Y, Li F, et al. Fruit and vegetable consumption and the risk of depression: a meta-analysis. Nutrition 2016;32:296-302.

12. Blanchflower DG, Oswald AJ, Stewart-Brown S. Is psychological well-being linked to the consumption of fruit and vegetables? Soc Indic Res 2013;114:785-801.

13. McMartin SE, Jacka FN, Colman I. The association between fruit and vegetable consumption and mental health disorders: evidence from five waves of a national survey of Canadians. Prev Med 2013:56:225-30.

14. Richard A, Rohrmann S, Vandeleur CL, et al. Associations between fruit and vegetable consumption and psychological distress: results from a population-based study. BMC Psychiatry 2015;15:213.

15. Mihrshahi S, Dobson AJ, Mishra GD. Fruit and vegetable consumption and prevalence and incidence of depressive symptoms in mid-age women: results from the Australian longitudinal study on women's health. Eur J Clin Nutr 2015;69:585-91.

16. Gangwisch JE, Hale L, Garcia L, et al. High glycemic index diet as a risk factor for depression: analyses from the Women's Health Initiative. Am J Clin Nutr 2015;102:454-63.

17. Sánchez-Villegas A, Delgado-Rodríguez M, Alonso A, et al. Association of the Mediterranean dietary pattern with the incidence of depression. Arch Gen Psychiatry 2009:66:1090-8.

18. Hodge A, Almeida OP, English DR, et al. Patterns of dietary intake and psychological distress in older Australians: benefits not just from a Mediterranean diet. Int Psychogeriatr 2013;25:456-66.

19. Rienks J, Dobson AJ, Mishra GD. Mediterranean dietary pattern and prevalence and incidence of depressive symptoms in mid-aged women: results from a large community-based prospective study Eur J Clin Nutr 2013;67:75-82.

20. Jacka FN, Mykletun A, Berk M, et al. The association between habitual diet quality and the common mental disorders in community-dwelling adults: the Hordaland health study. Psychosom Med 2011;73:483-90.

21. Akbaraly TN, Brunner EJ, Ferrie JE, et al. Dietary pattern and depressive symptoms in middle age. Br J Psychiatry 2009;195:408-13.

22. Blazer DG. Depression in late life: review and commentary. J Gerontol A Biol Sci Med Sci 2003;58:249-65.

23. Banks E, Redman S, Jorm L, et al. Cohort profile: the 45 and up study. Int J Epidemiol 2008;37:941-7.

24. Andrews G, Slade T. Interpreting scores on the Kessler Psychological Distress Scale (K10). Aust N Z J Public Health 2001;25:494-7.

25. Rustihauser IHE, Webb K, Abraham B, et al. Evaluation of short dietary questions from the 1995 NNS. Canberra: Commonwealth Department of Health and Aged Care, 2011.

26. Australian Institute of Health and Welfare. The Active Australia Survey: a guide and manual for implementation, analysis and reporting. Canberra, ACT: Australian Institute of Health and Welfare, 2003.

27. Byles JE, Gallienne L, Blyth FM, et al. Relationship of age and gender to the prevalence and correlates of psychological distress in later life. Int Psychogeriatr 2012;24:1009-18.

28. Chi SH, Wang JY, Tsai AC. Combined association of leisure-time physical activity and fruit and vegetable consumption with depressive symptoms in older Taiwanese: results of a national cohort study. Geriatr Gerontol Int 2016;16:244-51.

29. Mujcic R, Oswald A. Evolution of well-being and happiness after increases in consumption of fruit and vegetables. Am J Public Health 2016;106:1504-10.

30. Rooney C, McKinley MC, Woodside JV. The potential role of fruit and vegetables in aspects of psychological well-being: a review of the literature and future directions. Proc Nutr Soc 2013;72:420-32.

31. Almeida OP, Ford AH, Flicker L. Systematic review and meta-analysis of randomized placebo-controlled trials of folate and vitamin B12 for depression. Int Psychogeriatr 2015;27: 727-37.

32. Bottiglieri T. Homocysteine and folate metabolism in depression. Prog Neuropsychopharmacol Biol Psychiatry 2005;29:1103-12.

33. Kingsbury M, Dupuis $G$, Jacka $F$, et al. Associations between fruit and vegetable consumption and depressive symptoms: evidence from a national Canadian longitudinal survey. J Epidemiol Community Health 2016;70:155-61. 


\section{Correction}

Nguyen B, Ding D, Mihrshahi S. Fruit and vegetable consumption and psychological distress: cross-sectional and longitudinal analyses based on a large Australian sample. BMJ Open 2017;7:e014201. doi: 10.1136/bmjopen-2016-014201

In Table 1, the "K10 score at baseline $\geq 22$ " column, row " $\leq 10$ years of education", the percentage should read $38.7 \%$ and not $68.1 \%$.

Open Access This is an Open Access article distributed in accordance with the Creative Commons Attribution Non Commercial (CC BY-NC 4.0) license, which permits others to distribute, remix, adapt, build upon this work non-commercially, and license their derivative works on different terms, provided the original work is properly cited and the use is non-commercial. See: http://creativecommons.org/licenses/by-nc/4.0/

BMJ Open 2017;7:e014201corr1. doi:10.1136/bmjopen-2016-014201corr1

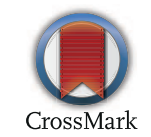

